\title{
Optimal Capacity Provisioning for Label Switched Paths in MPLS Networks
}

\author{
C. Bruni ${ }^{1}$, C. Scoglio ${ }^{2}$, and S. Vergari ${ }^{1}$ \\ 1 Dipartimento di Informatica e Sistemistica, University of Rome "La Sapienza", \\ Via Eudossiana 18, 00184 Rome, Italy \\ \{brunic, vergari\}@dis.uniroma1.it \\ 2 Broadband and Wireless Networking Lab, School of Electrical and Computer \\ Engineering, Georgia Institute of Technology, Atlanta GA 30332, USA \\ caterina@ece.gatech.edu
}

\begin{abstract}
Optimal control is a possible approach to Internet traffic engineering, which aims to achieve QoS guarantees and efficiency in network resources use. The goal can be better achieved by using the MultiProtocol Label Switching technique (MPLS), which provides increased scalability, manageability and enhanced QoS functions in IP-based networks. In this context, this paper proposes a method to find the optimal capacity provisioning for a Label Switched Path (LSP) of a MPLS network. The optimal capacity allocation for a given time interval is computed with respect to a quadratic cost function including a switching cost and a management cost for the whole network. The unique optimal solution is analytically computed assuming the knowledge of the offered traffic for the whole control interval. Furthermore, a sub-optimal on line solution is proposed which only requires the knowledge of a narrow sliding window of the offered traffic. Optimal and sub-optimal solutions are compared with respect to a simulated case study, enlightening the simplicity and, at the same time, the effectiveness of the second one.
\end{abstract}

\section{Introduction}

The growth of Internet has made evident the need for Traffic Engineering (TE) which became an essential tool for Internet Service Providers (ISPs) to optimize the network resources utilization in order to achieve QoS guarantees. Today, QoS-based services are offered in terms of contract agreements between the ISP and its customers.

Several architectures for supporting QoS have been developed 12 . A strategy proposed in IETF[3] is to consider two different types of services: Corporateservice and Customer-service.

Corporate-service is based on a contract, the Service Level Agreement (SLA), between the customer and the ISP, which is valid for a reasonable period of time and for a large amount of bandwidth. This type of agreement can be considered "quasi permanent $S L A$ " and it is particularly appropriate for companies who frequently require QoS guarantees and connections among distant network

E. Gregori et al. (Eds.): NETWORKING 2002, LNCS 2345, pp. 947958 2002.

(C) Springer-Verlag Berlin Heidelberg 2002 
nodes (e.g. branch offices). The contract could also consider "quasi on-demand" requests, i.e., bandwidth requests which can be satisfied within a determinate period of time and not immediately "on-demand".

On the other hand, customer-service is not based on quasi permanent SLAs but supports the setup of dynamic QoS sessions.

From the above considerations, it turns out that TE can be exercised on two time-scales depending on the service nature:

Long-term: the traffic requests are predicted on a wide interval (days-weeksmonths) by the existing long-term SLA contract;

Short-term: decisions are based on the observed state of the operational network on a short interval (minutes-hours).

Multi-Protocol Label Switching (MPLS) can be used to perform TE [4]. It has been shown that MPLS provides increased scalability, manageability and QoS functions to IP-based networks [5].

MPLS is the convergence of connection-oriented forwarding techniques and the Internet's routing protocols 67. MPLS directs the flow of IP packets along a predetermined path inside the network, called Label Switched Path (LSP). The main concept of MPLS is to pad a label on each packet. Packets are assigned a short fixed length label that summarize the destination, the precedence, QoS information and route.

The LSP setup problem has been approached in order to reduce the number of LSPs in the network and to get an optimal resources utilization [8]9]. Another important issue is the MPLS network dimensioning: the objective is to accommodate all expected demands without overloading any part of the network.

In this paper we consider two routers connected by a direct LSP and we formulate the LSP capacity dimensioning problem as an optimization problem. We will assume that, at each time, the bandwidth request between the two considered routers is completely satisfied, partly by the LSP direct connection and partly by an alternative IP connection (this latter if the LSP capacity is not enough to satisfy the request). First we assume to know the bandwidth requests between the two routers over the whole control time interval $[0, \mathrm{~T}]$. This is a quite unlikely assumption because it is not possible to exactly predict the traffic profile that will be offered to the network, due to the nature of IP traffic. However, we observe some properties of the optimal solution that allow us to reduce the need for the bandwidth request knowledge just to a sliding window over $[0, \mathrm{~T}]$, centered on the current time. In this case we obtain a sub-optimal, almost "on line", solution.

In the context of long-term TE and in particular of corporate-service agreements which consider "quasi on-demand" bandwidth requests, the knowledge of a narrow sliding window over $[0, \mathrm{~T}]$ is a much more likely assumption because an ahead booking can be considered. Moreover, the proposed solution is completely independent of any stochastic assumption on the Internet traffic demand behaviour.

In the next Section, we formulate the optimal LSP capacity provisioning problem with the assumption of knowing the offered traffic over the whole control 
interval. Then the solution is found with respect to a quadratic cost function and subjected to a physical lower bound constraint. In Section 3, an on line sub-optimal solution of the same problem is proposed, obtained by reducing the hypothesis on the bandwidth requests knowledge. In the same Section, an analysis has been performed about the approximation offered by the sub-optimal solution with respect to the optimal one. In Section 4, some concluding remarks are given with respect to a simulated case study; the results confirmed the high approximation level of the sub-optimal solution, together with relevant advantages related to the reduction in the required information.

\section{Optimal Solution for the Capacity Provisioning Problem}

Let us denote by $[0, \mathrm{~T}]$ the fixed control time interval over which we assume to know the bandwidth requests between two fixed routers. We consider an uniform discretization of $[0, \mathrm{~T}]$ and denote by $k=1, \ldots, N$, the discrete time variable. Furthermore, let $b(k)$ and $x(k)$ respectively denote the bandwidth request and the LSP capacity at time $k$. We assume that $b(k) \in[0, A], k=1, \ldots, N$, where $A$ denotes the bandwidth availability on the LSP. We can consider the LSP capacity like a simple linear discrete time dynamical system:

$$
x(k)=x(k-1)+\Delta(k), \quad k=1,2, \ldots, N
$$

where the initial state $x(0)=x_{0}$ is assumed known and positive and the control variable $\Delta(k)$ represents the capacity variation of the LSP at time $k$.

The LSP capacity is constrained by the following inequalities:

$$
x(k) \geq 0, \quad k=1,2, \ldots, N
$$

which has an obvious physical meaning.

For the above LSP capacity provisioning problem, we have defined a cost function in order to take into account the most relevant cost terms and, in the meantime, to get a handling mathematical formulation. In particular we consider the following cost terms:

- LSP cost: it takes into account the cost due to the reserved capacity used to forward packets in MPLS mode. This cost, at time $k$, is assumed proportional to the LSP capacity:

$$
J_{l}(k)=c_{l} \cdot x(k)
$$

where $c_{l}>0$ is the unitary cost for LSP capacity allocation.

- Excess cost: it takes mainly into account a cost due to packets switching performed in IP mode and their routing on an alternative path that occurs when the LSP capacity is less than the bandwidth request $(x(k)<b(k))$. Following the criteria that forwarding packets in MPLS mode is less expensive 
than IP mode, we assume the unitary cost coefficient $c_{e}$ for the bandwidth request not allocated on the $\mathrm{LSP}$, greater than $c_{l}$. To emphasize the advantage of MPLS techniques and to promote their utilization, we consider the above mentioned cost depending quadratically on the difference between the LSP capacity and the bandwidth request. On the other hand, it may happen that, at a generic time $k$, the LSP capacity is greater than the bandwidth request $(x(k)>b(k))$. In this case a certain amount of bandwidth is reserved without utilization: we have assumed to penalize this event with a cost depending quadratically on the amount of waste bandwidth. For simplicity we consider the coefficient per unit of waste bandwidth equal again to $c_{e}$. From the above consideration, at time $k$, we have the following excess cost term:

$$
J_{e}(k)=c_{e} \cdot[b(k)-x(k)]^{2}
$$

where, as already said, $c_{e}>c_{l}$.

- Dimensioning variation cost: it takes into account the LSP dimensioning variation cost. Each change of LSP capacity is charged in order to avoid too much wide LSP capacity re-dimensioning, which in turns affects the dimensioning of the other LSPs in the MPLS network. The same term can also take into account the so called signalling cost which occurs at each LSP capacity variation. The dimensioning variation cost at time $k$ is assumed to depend quadratically on the size variation of LSP capacity:

$$
J_{v}(k)=c_{v} \cdot \Delta^{2}(k)
$$

where $c_{v}>0$ is the unitary dimensioning variation cost of the LSP.

It follows that the total cost function in the control interval is:

$$
J_{t}=\sum_{k=1}^{N} c_{l} x(k)+\sum_{k=1}^{N} c_{e}[b(k)-x(k)]^{2}+\sum_{k=1}^{N} c_{v} \Delta^{2}(k)
$$

From (11), the cost function (6) can be rewritten as follows:

$$
\begin{aligned}
J_{t} & =c_{e} \sum_{k=1}^{N} b^{2}(k)+c_{l} \sum_{k=1}^{N} x(k)-2 c_{e} \sum_{k=1}^{N} b(k) x(k)+\left(c_{v}+c_{e}\right) x^{2}(N)+ \\
& +c_{v} x_{0}^{2}+\left(2 c_{v}+c_{e}\right) \sum_{k=1}^{N-1} x^{2}(k)-2 c_{v} \sum_{k=1}^{N-1} x(k+1) x(k)-2 c_{v} x_{0} x(1)
\end{aligned}
$$

Our aim is to minimize the total cost $J_{t}$ with respect to the variable $x(k)$, $k=1, . ., N$, in the presence of constraints (2). Let us note that the terms $c_{e}$. $\sum_{k=1}^{N} b^{2}(k)$ and $c_{v} \cdot x_{0}^{2}$ do not depend on $x(k), k=1, . ., N$, therefore we do not consider them in the cost minimization. We can, at this point, formulate the following quadratic programming problem. 
Problem 1. Find a global minimum for the cost function:

$$
J(x)=x^{T} \cdot H_{N} \cdot x+f^{T} \cdot x
$$

in the admissible set:

$$
D=\left\{x \in \mathbb{R}^{N}: \quad x \geq 0\right\}
$$

where $x$ and $f$ are the following $N$-vectors:

$$
x=\left(\begin{array}{c}
x(1) \\
\vdots \\
x(N)
\end{array}\right), \quad f=\left(\begin{array}{c}
c_{l}-2 c_{e} \cdot b(1)-2 c_{v} \cdot x_{0} \\
c_{l}-2 c_{e} \cdot b(2) \\
\vdots \\
c_{l}-2 c_{e} \cdot b(N)
\end{array}\right)
$$

and $H_{N}$ is the following $N \times N$ matrix:

$$
H_{N}=\left(\begin{array}{cccccc}
2 c_{v}+c_{e} & -c_{v} & 0 & . & 0 & 0 \\
-c_{v} & 2 c_{v}+c_{e}-c_{v} & . & 0 & 0 \\
0 & -c_{v} & \cdot & . & . & . \\
. & . & . & \cdot & -c_{v} & 0 \\
0 & 0 & \cdot & -c_{v} & 2 c_{v}+c_{e} & -c_{v} \\
0 & 0 & . & 0 & -c_{v} & c_{v}+c_{e}
\end{array}\right) .
$$

The matrix $H_{N}$ is definite positive, as can be easily proved by exploiting some results in [10], so that $J$ is strictly convex in $\mathbb{R}^{N}$.

The solution of Problem 1 is given in the following theorem.

Theorem 2. Assuming $c_{e}>c_{l}, b(k) \geq \frac{1}{2}, k=1, \ldots, N$, the unique solution of Problem 1 is:

$$
x^{o}=-\frac{1}{2} H_{N}^{-1} \cdot f
$$

Proof. Taking the strict convexity of $J$ into account, the unique global minimum of $J$ in $\mathbb{R}^{N}$ is the solution of the equation:

$$
\left(\frac{d J}{d x}\right)_{x^{o}}^{T}=2 H_{N} \cdot x^{o}+f=0
$$

that is (11). In order to prove that (11) is also the unique solution of Problem 1, we will verify that $x^{o} \in D$, that is $x^{o}(k) \geq 0, k=1, . ., N$. The generic component of $x^{o}$ is:

$$
x^{o}(k)=-\frac{1}{2} \sum_{j=1}^{N}\left(H_{N}^{-1}\right)_{k j} \cdot f(j) \quad k=1,2, \ldots, N
$$


By suitably handling a result given in [10] about the analytical expression of $H_{N}^{-1}$, we have:

$$
\left(H_{N}^{-1}\right)_{i j}=\frac{c_{v}^{|i-j|}}{\operatorname{det}\left\{H_{N}\right\}} \operatorname{det}\left\{H_{N-\max \{i, j\}}\right\} \operatorname{det}\left\{K_{\min \{i, j\}-1}\right\}
$$

where $H_{i}, i=1, \ldots, N$, is an $i \times i$ matrix defined according to (10) and $K_{i}$ is the following $i \times i$ matrix:

$$
K_{i}=\left(\begin{array}{ccccc}
2 c_{v}+c_{e} & -c_{v} & 0 & . & 0 \\
-c_{v} & 2 c_{v}+c_{e} & \cdot & . & \cdot \\
0 & . & \cdot & -c_{v} & 0 \\
\cdot & \cdot & -c_{v} & 2 c_{v}+c_{e} & -c_{v} \\
0 & . & 0 & -c_{v} & 2 c_{v}+c_{e}
\end{array}\right), \quad i=1,2, \ldots, N
$$

Noting that $H_{i}$ and $K_{i}, i=1, \ldots, N$, are definite positive matrices, as can be proved by exploiting again results in [10], the positivity of $\left(H_{N}^{-1}\right)_{k j}$ follows from (13) for $k, j=1, \ldots, N$. The positivity of $x^{o}(k), k=1, \ldots, N$, is then implied by the positivity of $-f(j), j=1, \ldots, N$. This, in turn, is an obvious consequence of the assumptions and of the positivity of $x_{0}$.

Remark 3. It is worth noting that the optimal solution $x^{o}(k)$, for each $k$, depends on all the samples $b(j), j=1, \ldots, N$, as it clearly results from (12).

\section{Sub-optimal on Line Solution}

Although the hypothesis of complete knowledge of Internet traffic demand on the control discrete time interval $[0, N]$ is partially supported by long-term TE framework, our aim, in this Section, is to reduce this hypothesis. Indeed, we will show that, for the particular structure of the inverse matrix $H_{N}^{-1}$, we can motivate a sub-optimal solution assuming to know just a narrow sliding window on the bandwidth profile, centered at the current time, much smaller than the total time interval $[0, N]$ considered before. As a consequence, the structure which characterizes the sub-optimal solution can be implemented "on line", while the optimal one is clearly "off line".

In order to analyze the behaviour of the suboptimal solution we are going to introduce, let us define the following parameter:

$$
\alpha_{h}=\max _{i, j:|i-j|=h}\left\{\left(H_{N}^{-1}\right)_{i j}\right\} \quad h=1,2, \ldots,(N-1) .
$$

The behaviour of the above parameter $\alpha_{h}$ has been numerically investigated for different values of $c_{v}, c_{e}, c_{l}$ and $N$. The analysis has pointed out a monotone decreasing behaviour of $\alpha_{h}$, as shown for instance in Fig.s 1, 2, 3.

Let us now give the definition of the sub-optimal solution for the Problem 1. 
Definition 4. For a fixed integer $N>1$, let be $M \leq 2 N-3$ a positive odd integer. We define the following sub-optimal solution:

$$
x^{s o}=-\frac{1}{2} P_{N M} \cdot f
$$

where $P_{N M}$ is the $M$-diagonal matrix of dimension $N \times N$ with entries:

$$
\left(P_{N M}\right)_{i j}=\left\{\begin{array}{cl}
\left(H_{N}^{-1}\right)_{i j} & \text { for } \quad|i-j|=0,1, \ldots, \frac{M-1}{2} \\
0 \quad \text { for } & |i-j|=\frac{M+1}{2}, \ldots,(N-1)
\end{array}\right.
$$

Remark 5. From (16) it is obvious that the generic component $x^{s o}(k), k=1, \ldots, N$, depends on a sliding window of no more than $M$ components $f(j)$ of $f$. This means that the suboptimal solution, at each time $k$, requires the knowledge of bandwidth requests on a sub-interval containing no more than $\frac{M-1}{2}$ future samples $b(j)$.

In order to verify that $x^{s o}$ is a good approximation of $x^{o}$, we introduce an upper bound on the error, which depends on $M$ and is sufficiently small when $M$ is suitably chosen. In fact, considering the norm $\|\cdot\|_{\infty}$ and recalling that $b(k) \leq A, k=1, \ldots, N$, from (9) it results:

$$
\|f\|_{\infty}=\max _{k=1, \ldots, N}\{|f(k)|\} \leq c_{l}+2 c_{e} A+2 c_{v} x_{0}=2 C .
$$

Therefore

$$
\begin{aligned}
\left\|x^{o}-x^{s o}\right\|_{\infty} & =\max _{k=1, . ., N}\left\{\left|x^{o}(k)-x^{s o}(k)\right|\right\}=\frac{1}{2}\left\|\left(H_{N}^{-1}-P_{N M}\right) f\right\|_{\infty} \leq \\
& \leq \frac{1}{2}\left\|\left(H_{N}^{-1}-P_{N M}\right)\right\|_{\infty} \cdot\|f\|_{\infty} \leq \\
& \leq C \max _{i, j=1, \ldots, N}\left\{\left|\left(H_{N}^{-1}\right)_{i j}-\left(P_{N M}\right)_{i j}\right|\right\} .
\end{aligned}
$$

From (13) and the definite positivity of $H_{i}, K_{i}$, we have $\left(H_{N}^{-1}\right)_{i j}>0, i, j=$ $1, \ldots, N$. Then, from (16), taking the definition (14) of $\alpha_{h}$ into account together with its monotonic property, it results:

$$
\begin{aligned}
\max _{i, j=1, \ldots N}\left\{\left|\left(H_{N}^{-1}\right)_{i j}-\left(P_{N M}\right)_{i j}\right|\right\} & =\max _{i, j:|i-j|=\frac{M+1}{2}, \ldots,(N-1)}\left\{\left(H_{N}^{-1}\right)_{i j}\right\}= \\
& =\max _{h=\frac{M+1}{2}, \ldots,(N-1)} \alpha_{h}=\alpha_{\frac{M+1}{2}} .
\end{aligned}
$$

Therefore we have:

$$
\left\|x^{o}-x^{s o}\right\|_{\infty} \leq C \alpha_{\frac{M+1}{2}}
$$

In order to analyze the approximation level given by (17), it is useful to observe that if we set:

$$
\frac{M+1}{2}=h
$$


when $M$ is an odd integer running from 1 to $(2 N-3), h$ assumes the values 1 , $2, \ldots,(N-1)$. Therefore we can rewrite (17) as follows:

$$
\left\|x^{o}-x^{s o}\right\|_{\infty} \leq C \alpha_{h}
$$

and analyze the approximation level by exploiting the behaviour of $\alpha_{h}$.

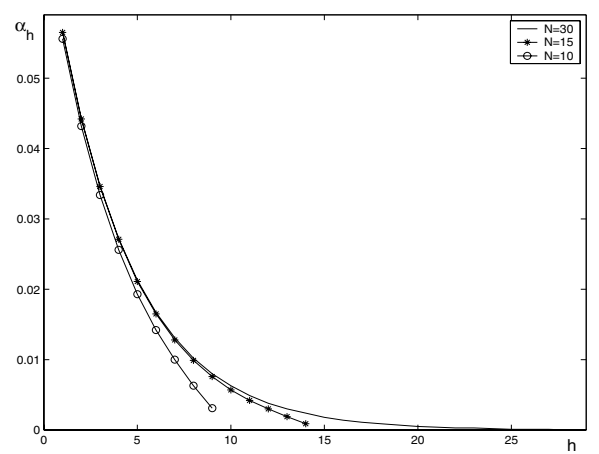

Fig. 1. Behaviour of $\alpha_{h}$ $\left(\mathrm{c}_{v}=50, \mathrm{c}_{e}=3, \mathrm{c}_{l}=1\right)$

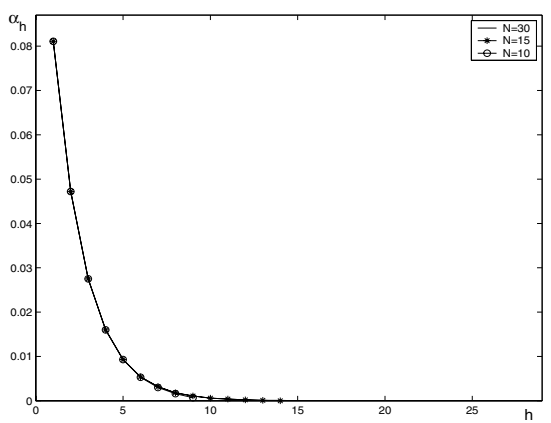

Fig. 2. Behaviour of $\alpha_{h}$ $\left(\mathrm{c}_{v}=10, \mathrm{c}_{e}=3, \mathrm{c}_{l}=1\right)$

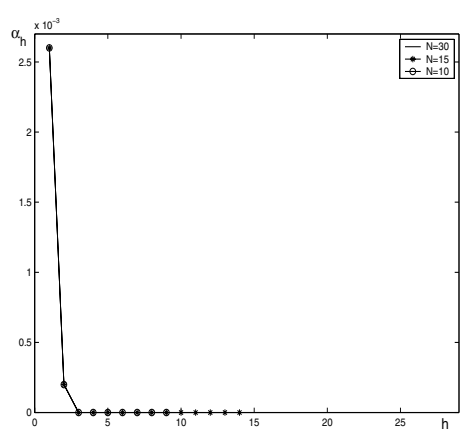

Fig. 3. Behaviour of $\alpha_{h}$ $\left(\mathrm{c}_{v}=3, \mathrm{c}_{e}=30, \mathrm{c}_{l}=1\right)$

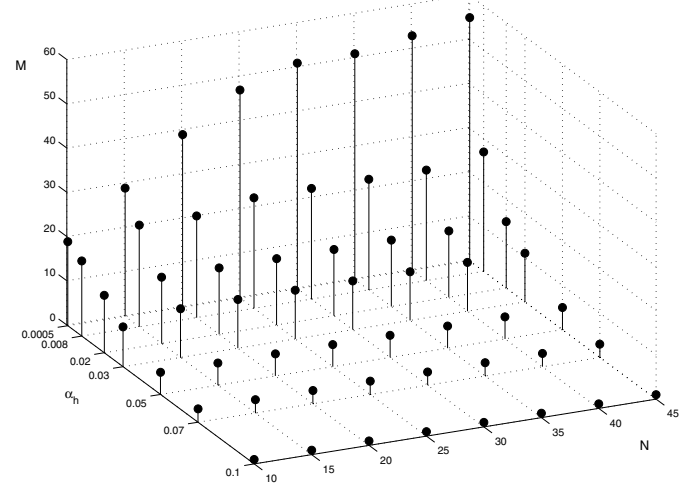

Fig. 4. Behaviour of $M$ $\left(\mathrm{c}_{v}=50, \mathrm{c}_{e}=3, \mathrm{c}_{l}=1\right)$

Remark 6. As it appears from Fig.s 1, 2, 3, $\alpha_{h}$ approaches quickly zero, for each fixed $N$, when $h$ reaches a few unit value. Therefore, also when $N$ increases, the approximation error can be kept low by assuming a suitable bounded value for $h$. For instance, in Fig. 1 and in Fig. 2 we have $\alpha_{h}<10^{-2}$ when $h \simeq 7$ (which amounts to the knowledge of only six future samples of $b(j))$ and this virtually for every $N$ greater than about ten. From Fig. 3, we observe that $\alpha_{h}<3 \cdot 10^{-3}$, for every $N$, also if $h$ is only equal to one (this means that $x^{s o}(k), k=1, \ldots, N$, 
depends only on the current request $b(k)$ and no knowledge of the future is required). The same conclusion is also evidenced by Fig. 4 where the behaviour of the parameter $M$, numerically obtained by (14) taking (18) into account, is also given in a 3-dimensional representation for different values of $\alpha_{h}$ and $N$, assuming for instance $c_{v}=50, c_{e}=3, c_{l}=1$. For $\alpha_{h}$ and $N$ fixed, Fig. 4 allows to deduce the corresponding value for the parameter $M$. It appears that $M$ quickly reaches a steady state value when $N$ increases, for each fixed $\alpha_{h}$.

\section{An Application to Simulated Data}

In order to test the application of the optimal and sub-optimal LSP capacity allocation procedures, we have considered a case study obtained by simulating a sequence of bandwidth requests.

To generate this bandwidth profile, we consider each request arrival time and each request death time as an event. Besides, we assume that two events occur at the same time with probability zero. In particular we simulate three stochastic processes:

- the first one generates the requests arrival times and it is simulated as a Poisson process with parameter $\lambda=\frac{1}{2}$;

- the second concerns the time duration of each request, and is characterized by an exponential distribution with parameter $\mu=\frac{1}{5}$;

- the last one is related to the amount of bandwidth of each request, and follows a uniform distribution on the integers of the interval $[1,10]$.

On the generated bandwidth profile we select a time window containing $N=$ 40 samples. As initial state we consider $x_{0}=11.3$ corresponding to the average value of the bandwidth requests. Using the above data, we compute the optimal and the sub-optimal solutions considering, for the parameters $c_{v}, c_{e}, c_{l}$, the same values as in Fig.s 1, 2, 3. We have considered a sub-optimal solution based, for instance, on the knowledge of only 8 future samples, which means $M=17$. Note that, for the choice $c_{v}=50, c_{e}=3, c_{l}=1$ and assuming $A=35$, Fig. 4 allows to guarantee an "a priori" approximation error with respect to the optimal solution not greater than 6.5 .

In Fig.s 5, 6, 7 computed optimal and sub-optimal solutions together with the simulated bandwidth profile are shown. In particular, from Fig. 5 we easily verify the above expected approximation level.

Concerning the effects of the cost parameters on the optimal solution, we have the following remarks:

- noting that the optimal solution is defined modulo a positive factor in the cost function, we have normalized the cost coefficients assuming always $c_{l}=$ 1 ;

- the parameter $c_{v}$, which weights the variation size cost, affects the behaviour of the optimal solution considerably; in particular the higher is the value of $c_{v}$, the smoother the solution becomes; 
- the parameter $c_{e}$ influences the fitting capability of the optimal solution with respect to the requested bandwidth reference. Note also that the same parameter influences the fitting capability of the sub-optimal solution with respect to the optimal one: this is due to the fact that, as $\frac{c_{e}}{c_{v}}$ increases, the matrix $H_{N}$ approaches the identity matrix and consequently $x^{s o}$ tends to coincide with $x^{o}$.

A comparison between the optimal and the sub-optimal solution can be carried out both with reference to the instantaneous approximation error and to the related costs. For the first point, we observe that the maximum deviation between $x^{o}$ and $x^{s o}$ is of about 3, 0.2, 0 respectively in the three considered cases. It is worth noting that virtually the same numerical results can be foreseen by exploiting the upper bound given by (17).

Concerning the related costs, considering for instance the first choice of parameters $\left(c_{v}=50, c_{e}=3, c_{l}=1\right)$, we obtain $J\left(x^{o}\right)=6672$, while for the sub-optimal solution (with $M=17$ ), we have $J\left(x^{s o}\right)=7141$, with an increase of about $6.56 \%$. It appears that the cost increase is very low, when compared with the advantage (in the better case) of discarding 31 future samples $b(j)$. Finally note that, if we want to furthermore reduce the cost increase, we can increase $M$; assuming for instance $M=21$ (10 future samples), the value of $J$ for the corresponding sub-optimal solution, becomes 6827 , which amounts to an increase of only $2.27 \%$.

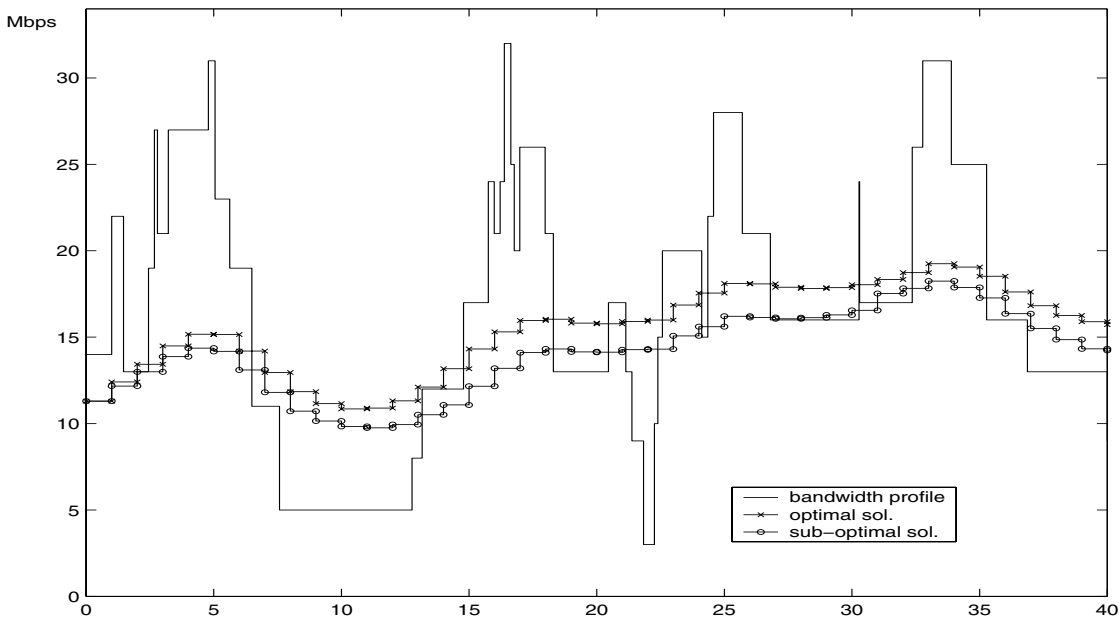

Fig. 5. Optimal and Sub-optimal solution $\left(c_{v}=50, c_{e}=3, c_{l}=1, M=17\right)$ 


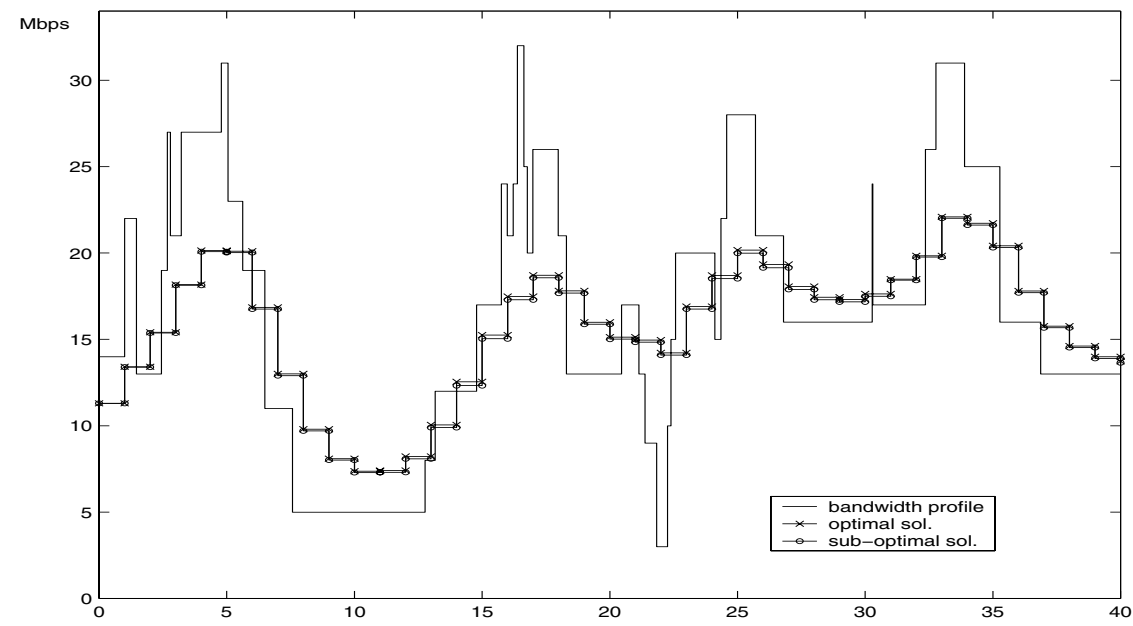

Fig. 6. Optimal and Sub-optimal solution $\left(c_{v}=10, c_{e}=3, c_{l}=1, M=17\right)$

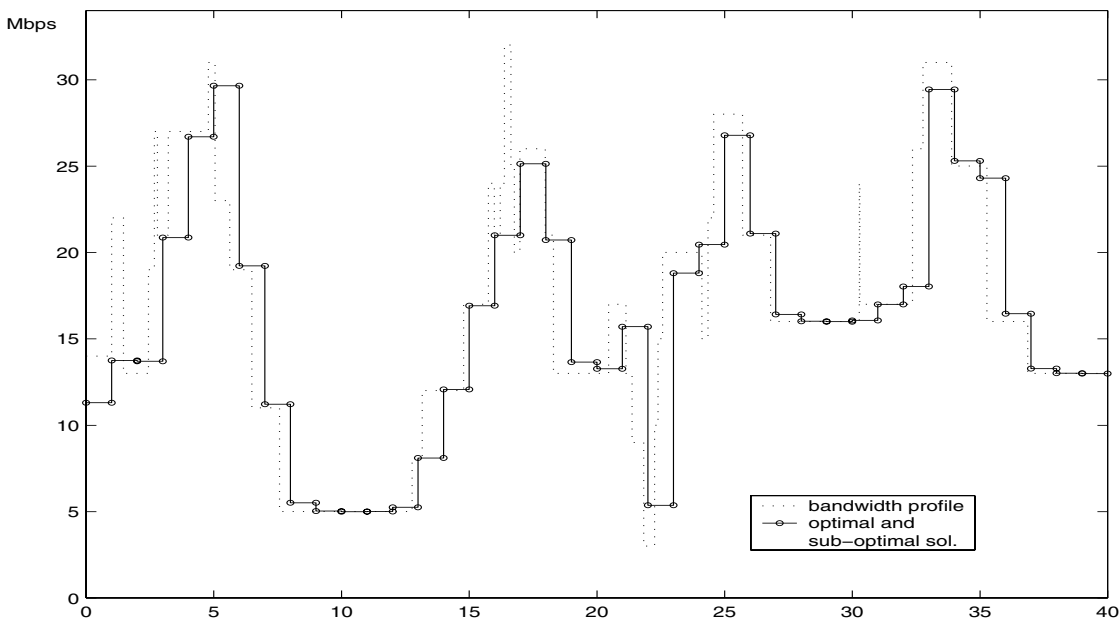

Fig. 7. Optimal and Sub-optimal solution $\left(c_{v}=3, c_{e}=30, c_{l}=1, M=17\right)$

\section{Concluding Remarks}

This paper provides a formal description of the optimal capacity provisioning problem for a label switched path in a MPLS network. In particular, by a suitable choice of the cost function, the above problem was reduced to a quadratic programming problem, whose closed form solution have been easily obtained.

This optimal solution requests the knowledge of all the future traffic in the control time interval. Being aware that future traffic knowledge is a quite unlikely assumption, by exploiting some properties of the optimal solution, we propose a sub-optimal one which offers the advantage of requiring the knowledge of future traffic only on a small sliding window over the control time interval and, at the 
same time, it offers a very good approximation level with respect to the optimal solution together with a very small increase of the cost.

Acknowledgments. The Authors are indebted to Professor I. F. Akyildiz for his encouragement and many valuable suggestions. Special thanks are also due to Professor F. Delli Priscoli for some useful discussions and to T. Anjali and J. C. Oliveira for their help in the realization of this paper.

\section{References}

[1] P. Trimintzios, D. Griffin, P. Georgatsos, D.Goderis, L.Georgiadis, C. Jacquenet, R. Egan: A Management and Control Architecture for Providing IP Differentiated Services in MPLS-Based Network. IEEE Communications Magazine, vol 39, n. 5, May 2001.

[2] R. Callon, E. Rosen, A. Viswanathan: MultiProtocol Label Switching Architecture. IETF, RFC 3031, January 2001.

[3] A. Bergsten, K. Nemeth, I. Cselenyi, G. Feher: Fundamental Questions Regarding End-to-End QoS. IETF, Internet Draft, July 2001.

[4] D. O. Awduche, J. Malcolm, J. Agogbua, M. O'Dell, J. McManus: Requirement for Traffic Engineering over MPLS. IETF, RFC 2702, September 1999.

[5] F. Gonzales, C. Chang, L. Chen, C. Lin: Using MultiProtocol Label Switching (MPLS) to Improve IP Network Traffic Engineering. Proc. Interdisciplinary Telecommunications Program, Spring 2000.

[6] G. J. Armitage: MPLS: the Magic Behind the Myths. IEEE Communications Magazine, vol 38, n. 1, Jan 2000.

[7] D. O. Awduche, A. Chiu, A. Elwalid, I. Widyaya, X. Xiao: A framework for Internet Traffic Engineering. IETF, Internet Draft, July 2001.

[8] C. Scoglio, T. Anjali, J. C. Oliveira, I. F. Akyildiz: A new Optimal Policy for Label Switched Path Setup in MPLS Network. Proc. 17th International Teletraffic Congress, Brazil, September 2001.

[9] H. Saito, Y. Miyao, M. Yoshida: Traffic Engineering Using Multiple Point-to-Point LSPs. Proc. INFOCOM 2000 (19th joint Conference of the IEEE Computer and Communication Societies), Tel Aviv, March 2000.

[10] C. F. Fischer, R. A. Usmani: Properties of some tridiagonal Matrices and their application to Boundary Value Problems. SIAM Journal on Numerical Analysis, Vol 6,n. 1, March 1969. 